\title{
Investigation of the velocity distribution in the flow of a journal bearing model
}

\author{
Matthias Nobis ${ }^{1 \mathrm{a}}$, Peter Reinke ${ }^{1}$, Marcus Schmidt ${ }^{1}$, Marco Riedel ${ }^{1}$ and Marcel Redlich ${ }^{1}$ \\ ${ }^{1}$ West Saxon University of applied sciences Zwickau, Dr.Friedrichs Ring 2a, 08056 Zwickau
}

\begin{abstract}
In many previous studies the main focus was put on the pressure distribution in the lubricating gap. Due to the limited space in the gap an investigation of the velocity distribution is very difficult or rather impossible. Based on the geometrical shapes of a real journal bearing, a bearing model test rig with an increased relative gap width has been developed. Thus, it is possible to detect the distribution of the flow speed within the gap by using a Laser Doppler Velocimeter (LDV). The comparability of the flow to the flow in a real journal bearing is ensured by observing the Reynolds similarity. Due to a targeted eccentricity in the system and the circumferential groove over $180^{\circ}$, there is in connection with the outlet hole in the rotating shaft a permanent change in the outflow conditions. The consequence is a periodically varying system pressure with effects to the pressure and volumetric flow rate at the inlet and outlet. The velocity measurements with a triggered LDV are done by considering these transient boundary conditions at the system boundaries. In this paper the experimental setup, the expiration of the investigations and some exemplary results are presented. Attendant to the experiment, numerical simulations are carried out and the results are compared with the experimental data.
\end{abstract}

\section{Introduction}

In multi-cylinder internal combustion engines with forged or casted crankshafts hydrodynamically lubricated journal bearings are used as the main bearings of the crankshaft. Such a main bearing is characterized by a feed hole for the oil supply, which opens into a groove in the bushing. To supply further bearings with fresh oil there are outlet holes and oil channels in the crankshaft, which link the main bearings with the bearings of the connecting rods. In the small lubrication gap between the rotating shaft and the stationary bushing there are threedimensional, laminar flow structures. Caused by the outflow of the oil through the outlet hole in the rotating crankshaft unsteady flow conditions result.

The experimental investigations are used as a reference for the development of new numerical computation algorithms. The aim is the formulation of a calculation tool, which is able to reproduce the threedimensional flow in the lubrication gap correctly and which predict areas of local vapour pressure. The base for such investigations are damages in bearings, which are caused by cavitation. The exact process of the cavitation bubble formation in the small lubricating gap is still not known sufficiently. Current commercial simulation programs are based on a two-dimensional calculation

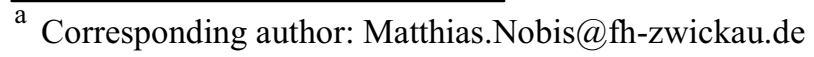

approach. The gap thickness in the radial direction is neglected.

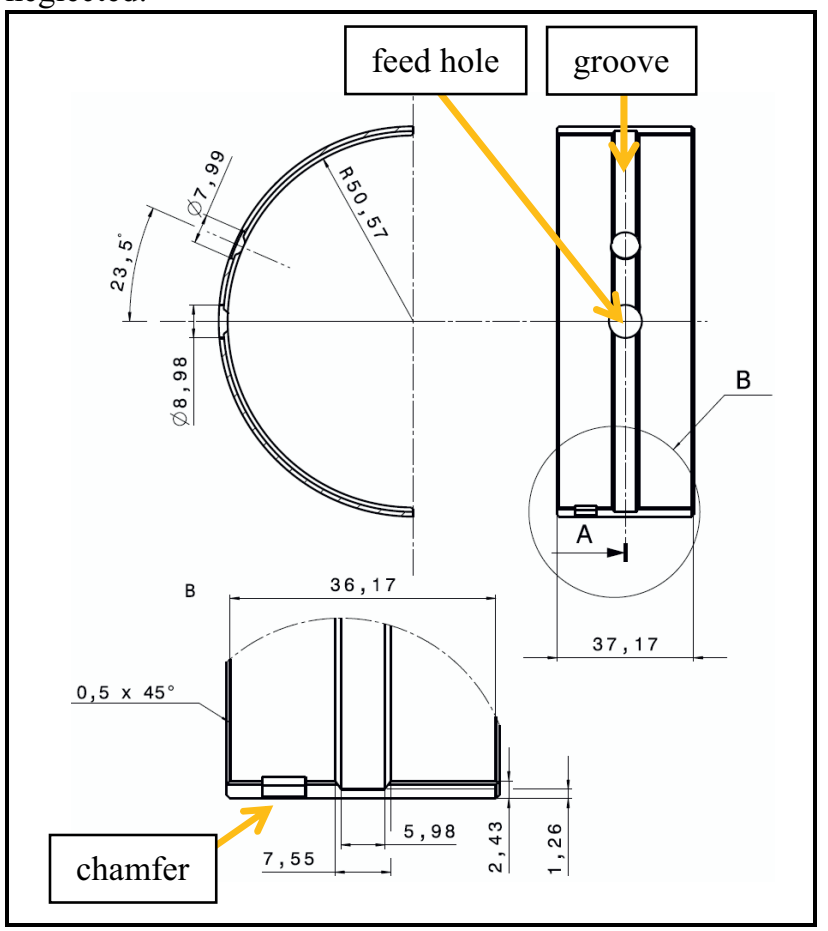

Figure 1. Bushing of a crankshaft main bearing 
Thus, if there is a local vapour pressure area, it is impossible to locate the vapour bubbles exactly and to distinguish between the shaft and the bearing shell. In order to make precise statements about the causes of formation of vapour bubbles in the lubricating gap, the system must be considered in three dimensions.

The determination for the bushing geometry shown in Figure 1 is based on the experimental tests of Wollfarth [1]. In this work the affinity of the bearing to cavitation was investigated under a variation of parameters such as Reynolds number, bearing load and the amount of supplied oil. Moreover different combinations of shafts and bearing shells were investigated in terms of cavitation. Only in a few cases it was possible to generate cavitation repeatable. The bearing shell in Figure 1 has the geometrical shapes of such a bearing, which tends to reproducible cavitation.

\section{Geometrical parameters of the model}

Figure 2 shows a schematic longitudinal and transverse section through the bearing model. The angle $\varphi=0$ is defined as the widest gap. Starting from the widest gap the feed hole has an angular position phi.B. Similarly, the measurement position is also indicated by an angular position. The breadth of the groove and the diameters of the feed hole respectively the outlet hole are identical. In the equations 1 to 6 characteristic parameters are defined.



Figure 2. Geometrical parameters

$$
\begin{gathered}
H_{0}=R_{2}-R_{1} \\
\Psi=\frac{H_{0}}{R_{1}} \\
U_{1}=\omega \cdot R_{1} \\
R e=\frac{H_{0} \cdot R_{1} \cdot \omega}{v} \\
\varepsilon=\frac{e}{H_{0}} \\
Q_{0}=0,5 \cdot B \cdot H_{0} \cdot U_{1}
\end{gathered}
$$

\section{Test rig}

The geometrical characteristics of the bearing system shown in Figure 1 are transferred to the bearing model test rig in a scale of $3: 1$. The groove is simplified to a rectangular profile without any bevels. Furthermore, the chamfers, which secure the bearing shell against rotation, will be unconsidered. In order to provide the optical access for the LDV to the lubrication gap, the relative gap width is significantly increased in comparison to the original journal bearing.



Figure 3. Experimental setup

The bearing model test rig has a relative gap width of $\psi \approx 2.5 \%$. In contrast, real bearings have a relative gap width of approximately $\psi \approx 0.1 \%$. Nevertheless with the 
compliance of the Reynolds similarity the analogy to the flow in a real journal bearing is ensured. The oil supply to the bearing model is realized with the feed hole in the fixed outer cylinder. The outflow of the oil is carried out via the outlet hole in the rotating inner cylinder. By using a hollow drilled rotating shaft and a rotary union the oil returns to the reservoir.

The connected hydraulic circuit consists of a controllable pump, a control valve and an ultrasonic flow meter with a measuring range from $3 \mathrm{l} / \mathrm{min}$ to $36 \mathrm{l} / \mathrm{min}$ for an exact measurement of the applied oil flow rate (see Figure 3). The outer cylinder can be mounted in an eccentric position relative to the inner cylinder. For this purpose, it is fixed with clamps on a plane turntable. By the arrangement on a rotary table, there will be the possibility to perform speed measurements at different angular positions without traversing the LDV on a circular path. The exact radial and axial positioning of the measurement volume is done by using an X-Y-traverse system.

The pressure difference between the inlet and outlet is measured by a differential pressure sensor with a measuring range from 0 mbar to $250 \mathrm{mbar}$. For a precise correlation of the recorded measurement values to the rotational angle of the inner cylinder, there is a light barrier fitted at the drive unit of the test bench. This light barrier generates with each rotation of the inner cylinder a pulse at an angle of $0^{\circ}$. This signal is used as a reset pulse for the signal processor of the LDV and to refer the difference pressure and volumetric flow rate measurements to a rotational angle.

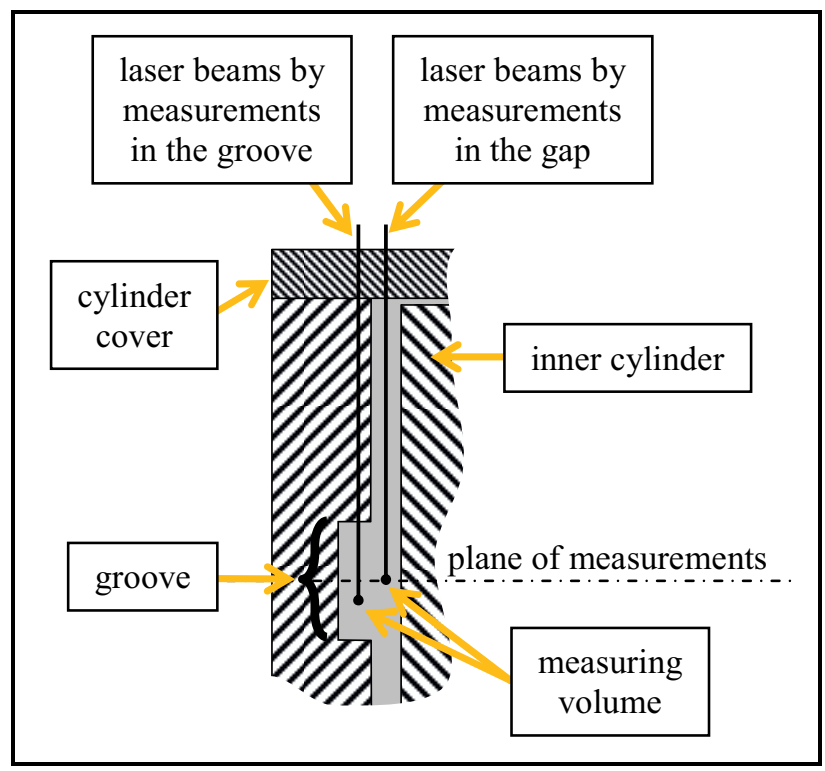

Figure 4. Path of the laser beams by measuring in the groove and by measuring in the gap

If the measuring volume of the LDV is positioned in the gap, then the laser beams penetrate $10 \mathrm{~mm}$ of and $57.5 \mathrm{~mm}$ of fluid. For velocity measurements in the groove the rays penetrate $55.5 \mathrm{~mm}$ of acrylic glass and $12 \mathrm{~mm}$ of fluid (see Figure 4). The different refractive indexes of this two mediums cause a shift of the measuring volume. For velocity measurements directly in the groove it is necessary to traverse the LDV in the axial (vertical) direction to compensate the refractive processes. In the first step and as part of this publication all measurements are done in the mid plane $(Z=0)$ of the system (see Figure 2).

\section{Numerical model}

The numerical simulations were performed using the software OpenFOAM. The code is based on the finite volume method and uses the three-dimensional, incompressible Navier-Stokes-Equations and the mass balance equation. In Figure 5 the volume mesh of the bearing model is shown. The model is built by a blockstructured mesh with nearly orthogonal cells in the gap. With this meshing method every cell has a unique relationship to the next cell neighbour and increases consequently the efficiency of the numerical solver. For the feed hole as well as the outlet hole a typical O-grid with wall adapted cells is used.

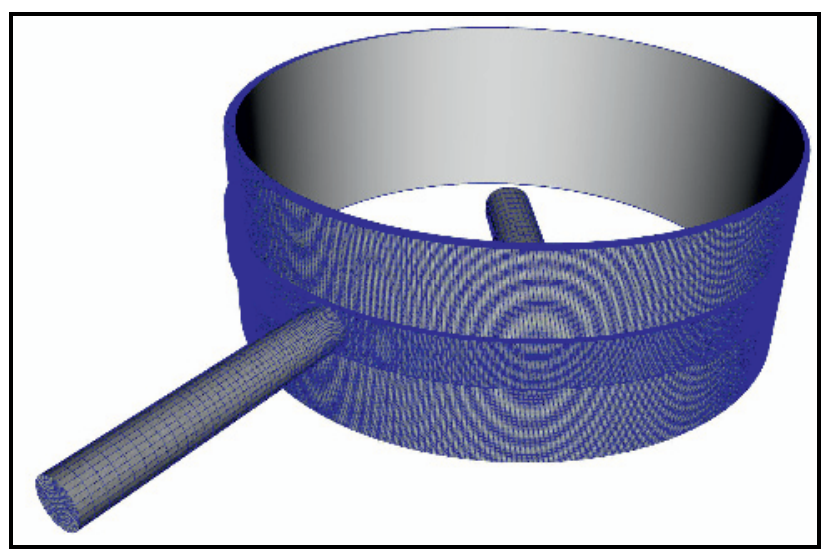

Figure 5. Volume mesh of the numerical model

\section{Results of the researches}

Due to the outflow of the applied oil through the outlet hole in the rotating inner cylinder, there are transient operating conditions. An unsteady flow results not only directly in the lubricating gap. The pressure and volumetric flow rate in the peripheral circuit are also subject to periodic fluctuations. For a comparison between numerical and experimental data it is necessary, to pay particular attention to the proper definition of the boundary conditions. It is not effective, to set fixed boundary conditions with averaged values for the volumetric flow rate at the inlet and outlet. For an exact reproduction of the real flow by using CFD, time-varying boundary conditions must be defined.

The feed hole respectively the outlet hole in the numerical model are generated up to a specified length (see Figure 5). Each end of these holes is a system boundary, where the conditions for pressure and volumetric flow rate have to be defined. The volume mesh pretends the positions for the difference pressure measurements in the experiment. The input data for these boundary conditions are provided by the experimental pressure and flow rate measurements. 
Figure 6 shows the measured pressure difference between the inlet and outlet as well as the volumetric supply flow rate over one revolution of the inner cylinder.



Figure 6. Applied volumetric flow rate $Q_{i n}$ and resulting pressure difference between inlet and outlet for $\psi=2,5 \%$, $R e=35, \varepsilon=80 \%, \varphi_{B}=0^{\circ}$

At a rotational angle of $180^{\circ}$, the outlet hole of the inner cylinder is located in the narrowest gap. Caused by the small local gap width there is an enormously reduced effective outlet section. In consequence, a maximum pressure drop and a minimum volumetric flow rate should be expected at a rotational angle of $180^{\circ}$. As Figure 6 illustrates, there is obviously a shift of the two extremes into the direction of a rotational angle of $270^{\circ}$. This phase shift is caused by the inertia of the fluid flowing in the peripheral hydraulic circuit.

Figure 7 shows a comparison of one chosen experimental and numerical velocity profile under the given setting parameters in a scaled diagram. These profiles are detected at an angular position of $\varphi=8.96^{\circ}$. This diagram can be understand as a snapshot of a transient flow at a rotational angle of the inner cylinder of $40^{\circ}$. The corresponding values for the pressure difference between the inlet and outlet as well as the applied volumetric flow rate according to the angular position can be seen in Figure 6.

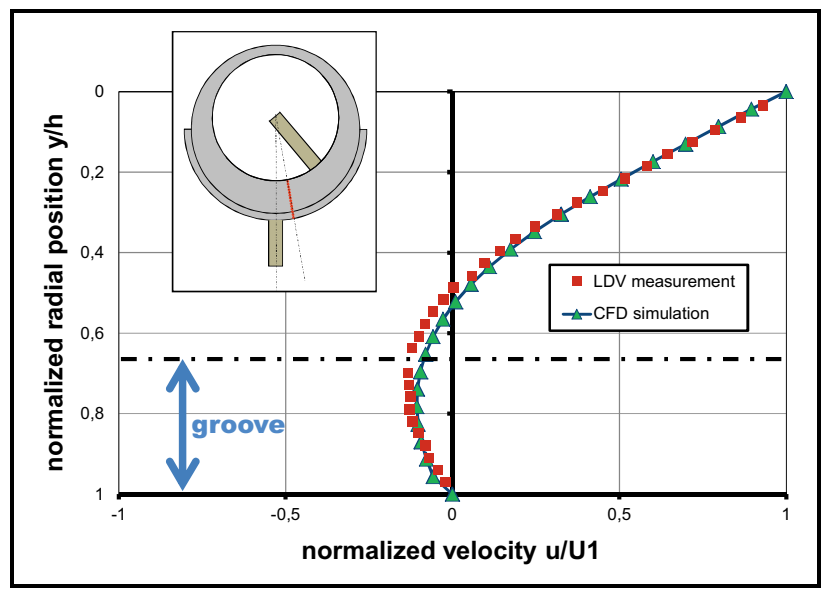

Figure 7. Velocity profiles of a LDV measurement and a CFD simulation at a rotational angle of the inner cylinder of $40^{\circ}$ for $\psi=2,5 \%, \operatorname{Re}=35, \varepsilon=80 \%, \varphi_{B}=0^{\circ}, \varphi=8,96^{\circ}$

The measured velocities are phase averaged over a rotational angle of one degree. Consequently, the LDV system delivers 360 measurement values per one revolution. Regarding to the adhesion between the oil and the walls of the system, the normalized velocity U/U1 is direct at the surface of the inner cylinder $(y / h=0)$ exactly one. Accordingly the normalized velocity is zero at the surface of the stationary outer cylinder $(y / h=1)$. At the transition from the lubrication gap to the area of the groove, it is impossible to measure reliable velocities, caused by the undefined refraction of the laser beams at the edges of the groove. Hence, there are two missing values at the corresponding radial position in the measured velocity profiles. However, the experimentally and numerically determined velocity profiles can be compared well. As Figure 7 shows, a good agreement between the results is achieved.

The three-dimensional CFD simulation can provide a more global view of the flow field in the lubricating gap. Figure 8 shows a streamline plot under the given boundary conditions. The rotating direction of the inner cylinder is anti-clockwise. The starting point of all streamlines is located at the inlet of the feed hole. The incoming fluid is distributed into all directions in the gap. A significant amount of supplied oil flows with the rotating direction of the inner cylinder directly into the outlet hole.

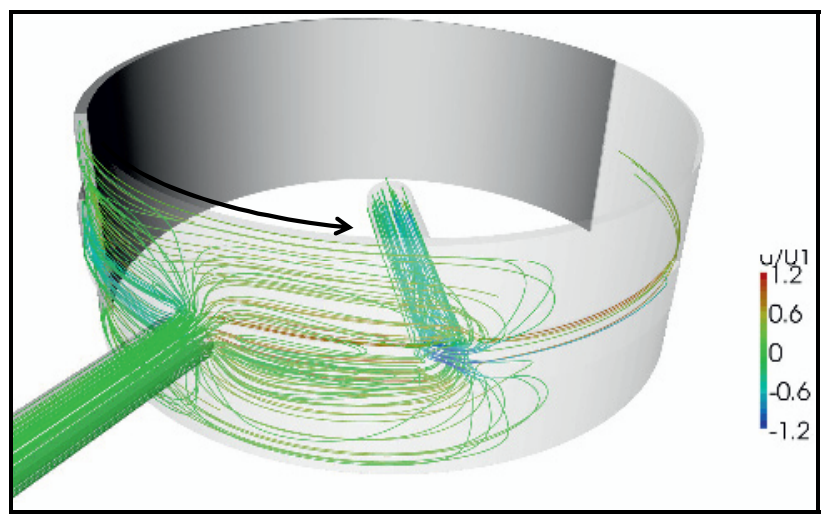

Figure 8. Streamline plot at a rotational angle of the inner cylinder of $40^{\circ}$ for $\psi=2,5 \%, R e=35, \varepsilon=80 \%, \varphi_{B}=0^{\circ}$

In an angular range from approximately $5^{\circ}$ to $14^{\circ}$ and by an adjusted measurement position of $\varphi=8.96^{\circ}$, the outlet hole of the inner cylinder is located directly at the current position of the measuring volume (see schematic illustration in Figure 9). Accordingly, for this angle range the no-slip condition to the surface of the inner cylinder is not valid. Consequently, the normalized velocities at the surface of the inner cylinder $(y / h=0)$ are not necessarily one. Figure 9 shows the velocity profile at a rotating angle of $7^{\circ}$ under the given boundary conditions. Remarkable is the clearly pronounced backflow area over the major part of the gap and across the whole groove. At this rotational angle, the center of the outlet hole is still in front of the actual measurement location. In the 
immediate vicinity of the outlet hole there is at all times a flow towards the outlet. Consequently, there is a reverse of the flow, when the outlet hole is located just before the measurement position.

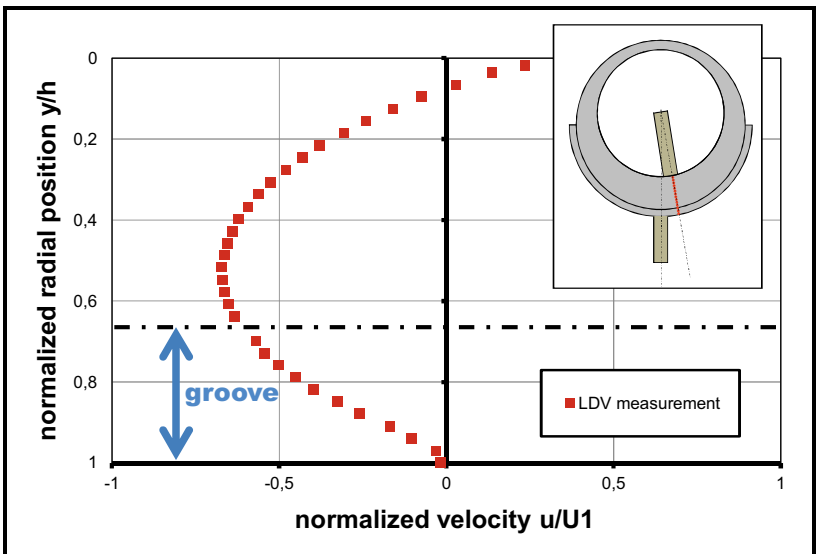

Figure 9. Velocity profile of a LDV measurement at a rotational angle of the inner cylinder of $7^{\circ}$ for $\psi=2,5 \%$, $\operatorname{Re}=35, \varepsilon=80 \%, \varphi_{B}=0^{\circ}, \varphi=8,96^{\circ}$

\section{Summary and Outlook}

The three-dimensional flow simulation delivers consistent results for all relevant state variables in the lubrication gap of the bearing model. However, for the optimization of the computational grid and to validate the numerical solver, it is absolutely necessary, to perform a comparison with experimental measurements. The velocity measurements using the LDV are a good way to create a base for comparisons between experiment and simulation.

The flow field in journal bearings is very complex with three-dimensional structures. There are not only transient conditions direct in the lubricating gap. The pressure and the volumetric flow rate at the system boundaries are also subject of periodic fluctuations. To quantify these fluctuations, the differential pressure between the inlet and outlet and the supplied volumetric flow rate were measured depending on the rotational angle.

Until now, only at one selected angular position velocity measurements were carried out. In further works, it is important to vary parameters such as eccentricity, applied volumetric flow rate and Reynolds number. Moreover, velocity measurements at further angular positions are necessary in order to get a more global overview over the flow in the lubricating gap on experimental side. Thus, there will be a more detailed data base to validate the numerical simulation.
$R_{1} \quad$ radius inner cylinder (shaft)

$R_{2} \quad$ radius outer cylinder (bushing)

Re Reynolds number

$u \quad$ circumferential speed

$U_{l} \quad$ circumferential speed of the inner cylinder

$\psi \quad$ relative gap width

$\varepsilon \quad$ relative eccentricity

$v \quad$ kinematic viscosity

$\varphi \quad$ angle

$\varphi_{B} \quad$ angle of the feedhole position

$\omega$ angular velocity of the inner cylinder

\section{References}

1. M. Wollfarth, Experimentelle Untersuchungen der Kavitationserosion im Gleitlager, (1995)

2. M. Nobis, P. Stücke, M. Schmidt, C. Egbers, N. Scurtu, Untersuchung der Zylinderspalt-strömung unter Berücksichtigung der Geometrieformen realer Gleitlager, 19. GALA conference, (2011)

3. G.I. Taylor, Stability of a viscous liquid contained between two rotating cylinders, (1923)

4. R.C. DiPrima, H.I. Swinney, Instabilities and Transistion in Flow Between Two Rotating Cylinders, in Hydrodynamic Instabilities and Transistion to Turbulence, (1985)

5. P.M. Eagles, J.T. Stuart, R.C. DiPrima, The Effects of Eccentricity on Torque and Load in Taylor-Vortex Flow, (1978)

6. E.L. Koschmieder, Taylor vortices between eccentric cylinders, (1976)

7. N. Scurtu, P. Stücke, C. Egbers, Numerical and experimental study of the flow in an eccentric Couette-Taylor system with small gap, PAMMconference, Appl. Math. Mech. vol. 8(1), 1064110642 (2008)

\section{Nomenclature}

$B$ nominal bearing width

e eccentricity

$H_{0} \quad$ nominal gap width

$h \quad$ local gap width

$Q_{0} \quad$ inner volumetric flow rate

$Q_{\text {in }}$ supply flow rate 